\title{
Minimally Invasive Mitral Valve Repair in a Marfan Patient with Severe Scoliokyphosis
}

\author{
${ }^{1}$ Department of Cardiac Surgery, Heart Center Leipzig University, \\ Leipzig, Germany \\ 2 Department of Radiology, Heart Center Leipzig University, \\ Leipzig, Germany \\ Thorac Cardiovasc Surg Rep 2014;3:1-2.
}

Thilo Noack ${ }^{1}$ Lukas Lehmkuhl ${ }^{2}$ Joerg Seeburger ${ }^{1}$ Friedrich Wilhelm Mohr ${ }^{1}$

Address for correspondence Thilo Noack, MD, Department of Cardiac Surgery, Heart Center Leipzig University, Struempellstrasse 39, Leipzig 04289, Germany (e-mail: thilo.noack@med.uni-leipzig.de).
Abstract
Keywords
- mitral valve regurgitation
- minimally invasive mitral valve repair
- minithoracotomy
- scoliokyphosis

A 26-year-old female Marfan patient with extensive scoliokyphosis presented with severe mitral valve regurgitation. The patient was treated with minimally invasive mitral valve repair via a right lateral minithoracotomy. In this report, we discuss the operative procedure followed in this special case and the current literature.

\section{Introduction}

The treatment of choice for mitral valve regurgitation is surgery. In recent years, the minimally invasive procedure via a right lateral minithoracotomy with femoral catheterization has become established. However, in patients with thorax deformations, the access via right lateral minithoracotomy remains a highly challenging clinical situation. In this report, we discuss a minimally invasive mitral valve (MV) repair via right lateral minithoracotomy in a Marfan patient with severe scoliokyphosis.

\section{Case Report}

A 26-year-old female Marfan patient presented with dyspnea (New York Heart Association functional class III-IV symptoms). Her medical history included multiple operations for severe leftright convection thoracolumbar scoliokyphosis ( - Fig. 1), coagulation disorder (von Willebrand disease), sleep apnea syndrome, and allergic reaction to antibiotics. Echocardiography revealed severe mitral regurgitation (MR) due to bileaflet prolapse and annulus dilatation. Left ventricular function was normal. Respiratory capacity was severely reduced to $0.84 \mathrm{~L}$. For preoperative planning, a computed tomography (CT) of thorax was performed to define the optimal access side from the chest wall to the MV apparatus (-Fig. 2).

received

October 10, 2013

accepted after revision

November 28, 2013

published online

February 27, 2014
The patient underwent minimally invasive MV surgery with a right minithoracotomy in the fourth intercostal space and femoral cannulation for cardiopulmonary bypass (CPB). ${ }^{1}$ She was placed on CPB prior to opening the pleura. A soft tissue retractor was utilized. A video camera was inserted through a $10-\mathrm{mm}$ port in the right third intercostal space and a transthoracic Chitwood aortic cross-clamp (Scanlan International, Inc., St. Paul, Minnesota, United States) was used for aortic occlusion. Antegrade crystalloid Bretschneider cardioplegia $(2,000 \mathrm{~mL})$ was administered directly into the aortic root. The left atrium was opened posterior to the interatrial groove. A left atrial retractor was used to expose the MV. MV repair was performed using two triangular resections on the posterior mitral leaflet between the P1 and P2 segment and between the P2 and P3 segment in combination with a sliding plasty as well as with edge-to-edge plasty on the A3/P3 level, and was completed using annuloplasty with a flexible annuloplasty band (Edwards Lifesciences, Irvine, California, United States). The total operating time was 189 minutes, the duration of CPB was 124 minutes, and the aortic cross-clamp time was 81 minutes. Intraoperative postrepair echocardiography showed normal MV function with no MR. There were no intraoperative complications and the patient had an uneventful recovery. At 6 months, the patient was observed to be in good health and free of cardiac symptoms as well as with no evidence of MR.
License terms

Stuttgart · New York

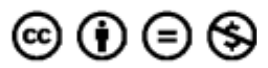




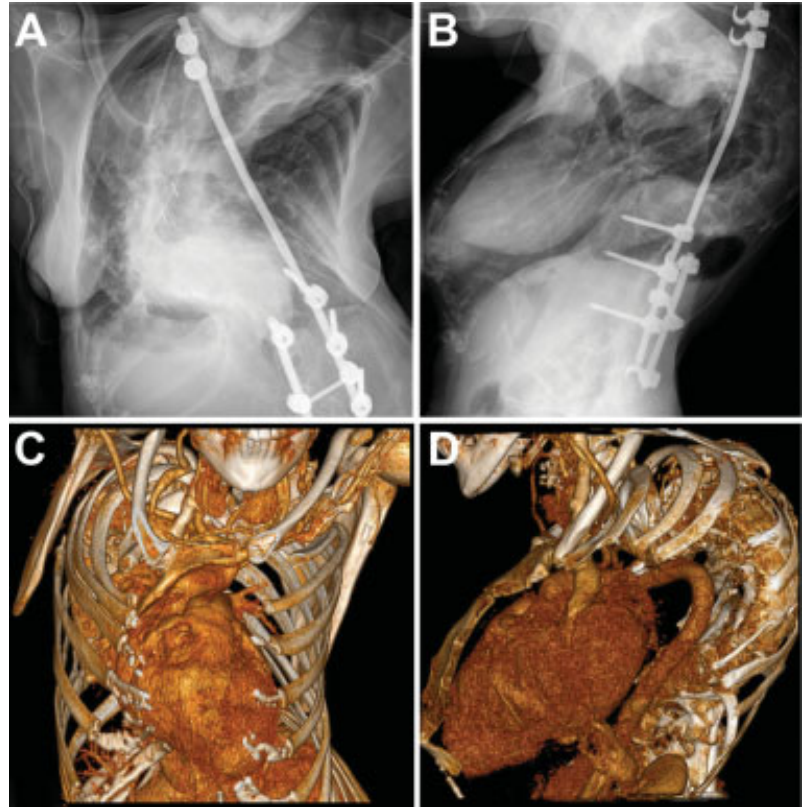

Fig. 1 (A) Posteroanterior and (B) lateral chest radiographs reveal extreme scoliokyphosis. The spine is stabilized by an internal fixation. Computed tomography images (three-dimensional reconstruction) showing $(C)$ right lateral minithoracotomy rout for the fourth intercostal space and (D) severe displacement of the spine, thorax, and heart.
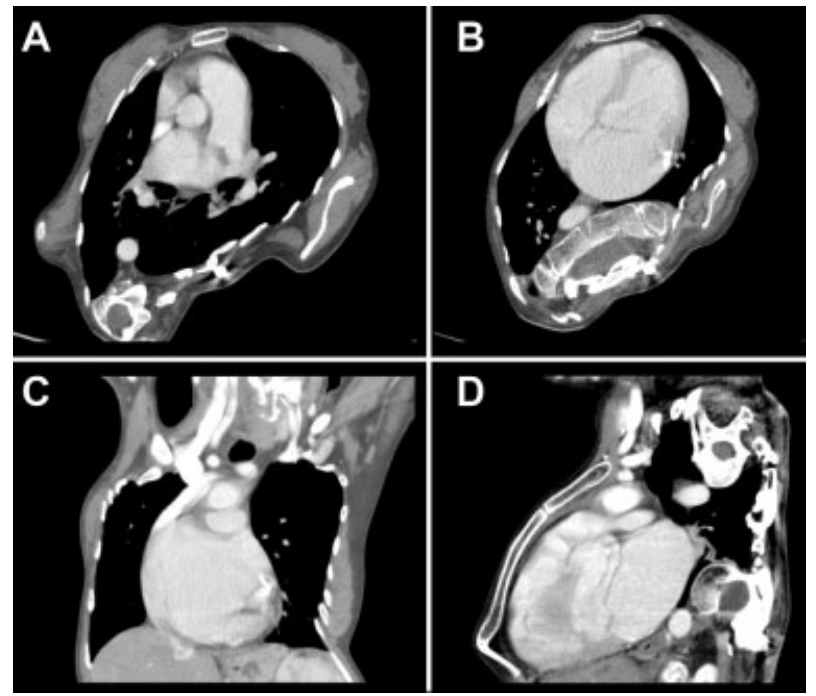

Fig. $2 \mathrm{CT}$ scan for preoperative planning showing (A) the torsion of spine and chest, (B) the possibility for a right lateral minithoracotomy in the fourth intercostal space, and (C-D) the anatomical relationship between heart, aorta, and sternum.

\section{Discussion}

This case is notable for (1) the severe spinal and chest deformity in the patient and (2) the minimally invasive access used in the deformed chest of the patient. To the best of our knowledge, this is the first such case in literature.

Marfan syndrome is an autosomal dominant disorder of connective tissue. Several disorders may be considered in patients with marfanoid features in their skeletal, cardiovascular, or ophthalmologic system. ${ }^{2}$ In the present case, the primary manifestation of Marfan syndrome was MR and severe thoracolumbar scoliokyphosis. ${ }^{3}$ MV dysfunction is the second most frequently accompanying cardiac lesion in Marfan syndrome. ${ }^{4}$ However, little is known about MV surgery in Marfan patients. ${ }^{5}$ Bozbuga et al reported nine Marfan patients with MR with a repair rate of $33 \%{ }^{4}$ They pointed out that the connective tissue defect might compromise the results of MV repair but emphasized on the importance of reconstructive surgery in young Marfan patients. von Kodolitsch and Robinson stated that results for MV repair in Marfan patients do not differ from those with myxomatous MV disease. ${ }^{2}$ This finding is underlined by Bhudia et al who showed an acceptable long-term durability of MV repair in Marfan patients. ${ }^{5} \mathrm{We}$ thus aimed for MV repair in this patient despite the heavily calcified MV annulus. Successful MV repair was achieved with modified classical triangular resection, edge-to-edge plasty, and annuloplasty.

Despite the extensive thoracolumbar scoliokyphosis and thus displacement of the heart with subsequent torsion of the cardiac axis, a median sternotomy seemed inappropriate compared with a minimally invasive right-sided approach (-Figs. 1 and 2). A median sternotomy most likely would have not been possible because of the remote and rotated position of the MV with an increased risk of cardiac injury. Thus, preoperative planning focused on the position of the heart and aorta within the chest with emphasize on the distance and angle of MV in relation to the lateral chest wall. With CT scan, we identified the fourth intercostal space as the best access site (-Fig. 2B). Intraoperative setting enabled excellent exposition of the MV and thus made the severe deformity of the thorax negligible. To our understanding, the torsion in this patient even eased the surgery from a technical point of view because the heart was directly underneath the chest wall.

The combination of Marfan syndrome, MR, and severe scoliokyphosis is a very rare but highly challenging clinical situation. The minimally invasive approach was highly suitable and contributed to successful MV repair due to excellent exposure.

\section{References}

1 Seeburger J, Borger MA, Falk V, et al. Minimal invasive mitral valve repair for mitral regurgitation: results of 1339 consecutive patients. Eur J Cardiothorac Surg 2008;34(4):760-765

2 von Kodolitsch Y, Robinson PN. Marfan syndrome: an update of genetics, medical and surgical management. Heart 2007;93(6): 755-760

3 Li QY, Qiu GX, Wang YP, et al. Clinical presentation and surgical treatment of scoliosis in Marfan syndrome. Chin Med J (Engl) 2005;118(15):1313-1317

4 Bozbuga N, Erentug V, Kirali K, Akinci E, Yakut C. Surgical management of mitral regurgitation in patients with Marfan syndrome. J Heart Valve Dis 2003;12(6):717-721

5 Bhudia SK, Troughton R, Lam BK, et al. Mitral valve surgery in the adult Marfan syndrome patient. Ann Thorac Surg 2006;81(3): 843-848 